\title{
Enabling Young Professionals to Learn from Errors - the Role of a Supportive Learning Climate in Crossing Help Network Boundaries
}

\author{
Therese Grohnert $^{1}$ (D) Roger H. G. Meuwissen ${ }^{2}$. \\ Wim H. Gijselaers ${ }^{1}$
}

Received: 17 July 2017 / Accepted: 8 May 2018 / Published online: 18 May 2018

(C) The Author(s) 2018

\begin{abstract}
Learning from errors is crucial for individuals' as well as organizations' performance. Yet, learning does not automatically follow from erring: the fear of negative consequences may prevent professionals from learning. These social costs of making an error, receiving negative judgments or experiencing feared punishment, need to be lowered for learning from error to take place. This study explores whether a supportive learning from error climate enables young professionals to engage in helpseeking behavior for learning after making an error. Using a social network approach, information from 157 young professionals in the domain of auditing reveals that not all help-seeking after errors is learning-oriented. Especially help-seeking from hierarchical superiors depends on the organization's learning from error climate: perceptions of this climate moderate the relationship between social costs of help seeking within a hierarchical work setting and the learning value of help sought. This study contributes to existing literature through its focus on a specific learning from error behavior, helpseeking, its triangulation of climate measures, as well as its focus on professionals' actual error experiences in their work environment.
\end{abstract}

Keywords Learning from errors · Help-seeking $\cdot$ Hierarchy $\cdot$ Accessibility $\cdot$ Learning from errors climate $\cdot$ Social network analysis

Therese Grohnert

t.grohnert@maastrichtuniversity.nl

1 Department of Educational Research and Development, School of Business and Economics, Maastricht University, P.O. BOX 616, 6200 MDMaastricht, The Netherlands

2 Department of Accounting and Information Management, School of Business and Economics, Maastricht University, P.O. BOX 616, 6200 MDMaastricht, The Netherlands 


\section{Introduction}

Making errors is inevitable (van Dyck et al. 2005; Rybowiak et al. 1999), especially when engaging in complex, professional tasks. Given possibly severe consequences of errors, such as the death of a hospital patient, a plane crashing, or the false reporting of financial information in auditing (Gold et al. 2014; Helmreich 2000; Tucker and Edmondson 2003), organizations have an inherent interest in managing the occurrence and the consequences of errors (Frese and Keith 2015). For the ones making errors, however, they are a double-edged sword; making errors is often seen as an indicator of poor ability, knowledge or performance, and at the same time, errors also provide unique opportunities for learning and development, especially in complex and changing environments (Edmondson 2011). Errors can be defined as "a deliberate action (or deliberate omission of actions) characterized by the unintended failure to achieve personal goals and/or the unintended deviation from organizational norms and goals which could have been avoided by alternative behaviors of the acting person" (Putz et al. 2012, p. 108). As a consequence, organizations wanting to benefit from learning from errors need to create an environment in which individuals can overcome the negative short-term connotations in order to engage in learning behavior after making an error (Bauer and Mulder 2013; Billett 2012; van Dyck et al. 2005).

Learning from errors requires a process of deriving new knowledge, new skills, and new methods from information provided by errors (Bauer and Mulder 2013; Billett 2012; Harteis et al. 2007; Zhao 2011). The concept of learning from errors has received most attention in the business and management literature when addressing learning at the organizational level (for a review, see Frese and Keith 2015). In contrast, less attention has been paid to individual learning from errors in work contexts (Bauer and Mulder 2013; Billett 2012). In the context of individual learning from errors, extant literature has focused on a variety of strategies and behaviors, such as fostering reflection on one's performance (Anseel et al. 2009; Hetzner et al. 2011), through decision-aids (e.g. Sibbald et al. 2013) and training with worked-out examples (Cattaneo and Boldrini 2017). The literature on social learning from errors has mostly emphasized the importance of communication as well as the enabling conditions and barriers to learning from others (Edmondson 2011; Frese and Keith 2015). Adding to this growing body of literature, the present study takes the individual as a starting point and focuses on errors as a trigger for learning in the social context of work (Eraut 2004, 2007; Marsick and Volpe 1999; Tynjälä 2013). This study focuses on a specific social learning behavior after occurrence of errors: seeking help within one's professional network. Bamberger (2009, p.52) defines help-seeking behavior as follows:

"for an action to classify as help-seeking, there must be (a) a problem or difficulty for which one hopes to find relief or some remedy, (b) at least two parties with one party soliciting the assistance of the other in what is fundamentally an interpersonal interaction, and (c) proactive action on the part of the help-seeker to gain the desired relief or remedy."

Focusing on help-seeking behavior as a means of learning from errors implies a behavior-focused perspective on an individual's actions after making an error with the specific intention to learn from their experience in a social work setting. Help-seeking 
literature has highlighted several barriers to effective help seeking for the purpose of learning. First, Borgatti and Cross (2003) focus on the concept of accessibility. This concept specifies that in order to be able to seek help, individuals need to know who possesses relevant knowledge and need to be able to actively contact these sources for any interaction to occur. Second, Bamberger (2009) highlights the role of emotional and social costs. High costs make it less likely that individuals will seek help, because they fear negative consequences. Encouraging learning from errors through helpseeking therefore requires the lowering of emotional and social costs. To summarize, accessibility is dependent on task and work allocation, while emotional and social costs are highest when help-seeking is perceived as a sign of incompetence, inferiority, or dependence (Bamberger 2009). Both barriers to help-seeking, accessibility and emotional and social costs, are therefore embedded in the social work environment and as such can be actively managed.

One characteristic of the social work environment in particular captures both barriers to help-seeking: hierarchical structures in organizations. Hierarchical superiors may be less accessible due to their organizational position, work commitments and / or physical distance. However, these superiors may possess relevant knowledge while at the same time evaluating performance (Bamberger 2009; van der Rijt et al. 2013). By consequence, hierarchical differences between help-seeker and help source may be associated with lower accessibility and higher emotional and social costs. The barriers inherent in hierarchical work structures affect one group in particular: young professionals at the bottom of the hierarchy. In their first working years, much of young professionals' attention is directed towards developing their professional knowledge, and by consequence, young professionals especially depend on seeking help in their social work environment (e.g. Wolfe Morrison 1993, 2012). Moreover, young professionals are prone to making errors based on their developing knowledge and shortage of work experience (e.g. Owhoso et al. 2002; Plumlee et al. 2015). By consequence, they are particularly vulnerable to the barriers to help-seeking in hierarchical settings: they depend on superiors' knowledge as well as on their assessment. Consequently, organizations wanting to encourage young professionals' learning from errors need to significantly mitigate the barriers to effective help-seeking.

While the barriers to learning from errors through help-seeking are inherent in the work environment, so is a mechanism to overcome them: individual professionals' beliefs about which behaviors are acceptable and desirable within a particular work environment. Putz et al. (2012, p. 112) define an organization's learning from errors climate as "the collective perceptions of the members of an organization or organizational unit concerning practices, processes, structures, and behaviors that support or hinder the benefit that organizations can draw from errors". A supportive learning from error climate is characterized by leader and colleague behavior conducive to undertaking social learning activities as well as by work processes that offer opportunities for learning from errors and sharing the resulting knowledge (Putz et al. 2012). In hierarchical work settings, norms and values are driven by leader behaviors: their actions may encourage social learning from errors or prevent it through sanctions (Edmondson 2011). Managers, supervisors, and unit leaders not only interpret decisions and norms from higher hierarchical levels, but they also create their own rules and standards for their workers which define the difference between right or wrong, error or doing the work correctly. Hierarchy, together with how managers and leaders accept 
drifting away from quality standards, and how workers deal with deviations from norms shape the social context of work. Studies across domains such as business, healthcare and education have found that social learning from errors takes place when facilitated through the firm's (learning from) error climate (Cannon and Edmondson 2005; Edmondson 2012; Tucker and Edmondson 2003; Zhao and Olivera 2006).

Adding to these findings, this study explores whether an organization's learning from error climate enables young professionals to overcome the barriers of accessibility and hierarchical boundaries, to enable learning from one's errors through help-seeking. In addressing this research question, the present study adds to existing literature in several ways. First, it uses a social network approach to measure professionals' proactive help-seeking behavior as an operationalization of learning from errors. This approach allows us to take into account professionals' actual social environment at the workplace. Second, it explicitly considers accessibility of valuable help and hierarchical boundaries as barriers to be overcome, combining help-seeking literature with learning from error literature. Third, we use a triangulation of climate measures to explore multiple facets of the influence of learning from error climate on individual help-seeking behavior. We investigate this research question in the setting of auditing, where professionals work in teams to verify whether financial statements of an organization fairly reflect the state of affairs. Young professionals in this field execute pre-structured tasks under the close supervision of a more experienced colleague. Teams have a strict hierarchical structure that is characterized by a layered system of reviews, an emphasis on efficiency and cost-awareness, and a high pressure to comply with standards set by regulators and overseers (Gronewold et al. 2013; Trotman et al. 2015).

\section{Theoretical Framework}

\section{Help-Seeking as Learning from Errors}

In operationalizing learning from errors for this study, we build on the workplace learning literature. Both Eraut (2007) and Marsick and Volpe (1999) specify that learning from experiences at the workplace occurs when triggered by a jolt, an event through which an individual recognizes that their current knowledge is insufficient to achieve a certain goal. Errors are a prime example of such a jolt (Frese and Keith 2015). In this study, we take as a starting point the moment in which an individual has recognized he or she made an error, has experienced the jolt, and we investigate if this jolt is followed up by the undertaking of a social learning activity - help-seeking (Bauer and Mulder 2013; Edmondson 2011). Bauer and Mulder (2013) and Frese and Keith (2015) emphasize that undertaking social learning activities after discovering one's own error is necessary when the individual cannot generate sufficient insight to learn from an error, e.g. through trial and error. This notion is also emphasized by the feedback literature, in which feedback by a knowledgeable person provides necessary information for performance improvement that the individual will not be able to realize without external input (Bamberger 2009; Shute 2008).

Within this social dimension of learning from errors, in this study, we focus on helpseeking as a social learning behavior after making and discovering one's own error in 
the workplace. Help seeking is often framed using a social network perspective, in which frequency and quality of interactions are considered between members of a certain group or unit, called ties (Borgatti and Cross 2003; Hatala 2006). An ego-centric social network takes an individual as the starting point and subsequently explores this person's social interactions with other individuals (ties) that form the person's network (Scott 2013). Following van der Rijt et al. (2013) and Cross and Sproull (2004), we focus on (a) the frequency with which young professionals seek help after making an error, and (b) the learning value of the help they receive on the specific error. Both concepts, frequency and learning value, relate to the literature on transactive memory, which explores how individuals come to know about their contacts' expertise (Lewis 2004; Van Der Kleij and Hoeppermans 2011; Wegner 1987). Knowing who to ask for help in a specific situation is a critical pre-condition for displaying the two learning behaviors that constitute the focus of the present study.

The social network perspective considers frequency of interactions as an expression of how acceptable a specific behavior is within a certain context, such as help-seeking (Hofmann et al. 2009; van der Rijt et al. 2013). The relationship between the frequency of help-seeking and outcomes such as (perceived) performance however is not straightforward. Prior research has shown a curvilinear relationship between help-seeking frequency and (perceived) performance (Nadler et al. 2003). The 'optimum' depends on the acceptability of help-seeking in the specific context (Bamberger 2009). We therefore assume that learning from error behavior is represented by frequent helpseeking, while allowing for a levelling-off effect. In addition to the frequency of helpseeking, social network analysis also allows operationalizing specific behaviors and motives in social interactions. In this study, we focus on the value of the help sought by young professionals with the specific purpose of learning from an error. Van der Rijt et al. (2013) operationalizes this learning value of help sought as targeted help-seeking from those ties in one's network whose possess relevant knowledge addressing a specific issue with the specific goal of learning. Indeed, the authors find a positive relationship between seeking help of high learning value and performance in the finance domain. We therefore assume that individuals who seek help of high learning value engage in learning from errors behavior.

\section{Social Barriers to Help-Seeking}

In the present study, we focus on the social barriers to young professionals' helpseeking; these social barriers apply once an individual decides to engage in social learning from errors (Bamberger 2009; Borgatti and Cross 2003; Hofmann et al. 2009). ${ }^{1}$ Two key barriers are identified in the respective bodies of literature, accessibility, and social/emotional costs incurred through relative hierarchy. The social network literature specifies accessibility as the key condition for any interaction to take place (Borgatti and Cross 2003; Hofmann et al. 2009). Accessibility is defined by Borgatti and Cross (2003) and Hofmann et al. (2009) as "the degree to which a potential help providers' expertise is available in a timely manner” (van der Rijt

\footnotetext{
${ }^{1}$ A discussion of individual characteristics and phenomena, such as personality and emotions, can be found in reviews by Frese and Keith (2015); and Metcalfe (2017) and in empirical work by e.g. Catino and Patriotta (2013) and Zhao (2011).
} 
et al. 2013, p. 263). Research on accessibility within social networks is rare in the learning (from error) literature. Van der Rijt et al. (2013) find that financial professionals who have access to knowledgeable colleagues in their professional network are more likely to seek help, seek help more frequently, and obtain help of higher quality. Similar evidence is available in the related fields of feedback- and information-seeking (Borgatti and Cross 2003; Hofmann et al. 2009). For help-seeking to lead to learning, individuals need to know which ties in their network possess relevant knowledge to be of use given a specific error (Wegner 1987).

Given this baseline condition of accessibility, the second concept, social / emotional cost, is discussed mostly in the help-seeking literature (Bamberger 2009). High social/ emotional costs occur when an individual is negatively judged, evaluated worse or even publicly shamed for making an error - these events lead to feelings of shame and incompetence (Bamberger 2009; Edmondson 2011). These emotions in turn may facilitate the hiding of errors or an unwillingness to help colleagues after making an error, preventing social learning from errors at the individual and the organizational level (Edmondson 2011; Rybowiak et al. 1999).

Both of these barriers, accessibility and social / emotional costs, are embodied in the concept of relative hierarchy (Bamberger 2009; van der Rijt et al. 2013). For example, easily accessible ties may not necessarily possess the most relevant knowledge to learn from errors. This view is addressed in the help-seeking literature, in which Bamberger (2009) finds that accessible ties are often similar to the individual seeking help, e.g. in hierarchy and knowledge. Seeking help from easily accessible ties may therefore be more frequent, due to higher accessibility, but may not represent learning behavior, due to similarities in knowledge and experience. Indeed, the opposite may be true: seeking help from a less accessible tie who possesses relevant knowledge is more likely to be undertaken for the sake of learning rather than for comfort (Bamberger 2009; Hofmann et al. 2009). At the same time, social/emotional costs are expected to be higher for ties at a higher hierarchical level, as superiors (a) establish desirable and undesirable behaviors with respect to errors (Edmondson 2004), and (b) supervise and evaluate the work of young professionals (Ashford and Cummings 1985; Lee 1997). And while more costly to obtain, help sought from hierarchical superiors may actually be more valuable due to superiors' advanced knowledge and experience (Bamberger 2009). This is however highly context-dependent: hierarchical superiors need to be familiar with the young professional's tasks and must be able to provide tailored help for it to be useful (Trotman et al. 2015). Empirically, this notion has been explored by van der Rijt et al. (2013), who find that financial professionals perceived help from hierarchical superiors to be more valuable for learning than help sought from hierarchical equals. Additionally, Tucker and Edmondson (2003) showed in the nursing setting that most interactions after making an error took place between professionals on the same function level. The authors interpret this behavior as emotional coping: seeking validation of one's feelings, but not new insights or additional knowledge to learn from an error, analogously to Hofmann et al.'s (2009) analysis of seeking help from similar contexts.

These notions thus lead us to distinguish between two patterns of help-seeking: coping and learning. Those young professionals who seek help frequently from easily accessible hierarchical equals are expected to engage in help-seeking behavior for the sake of coping with the jolt of making an error. In contrast, those young professionals who seek help of high learning value from hierarchical superiors irrespective of 
accessibility, are expected to engage in help-seeking behavior for the sake of learning, leading to the following hypotheses:

H1a: Young professionals will seek help more frequently from (1) easily accessible ties, and from (2) hierarchical equals.

$\mathrm{H} 1 \mathrm{~b}$ :Young professionals will seek help of higher learning value (1) independent of accessibility, and (2) from hierarchical superiors.

\section{Overcoming Social Barriers to Help-Seeking: an Organization's Learning from Error Climate}

Having specified two barriers to help-seeking, accessibility and emotional/social costs and the two patterns of help-seeking behavior to be distinguished (coping and learning), it is essential to understand how organizations can support young professionals in overcoming these barriers to encourage not just coping, but especially learning through help-seeking (Billett 2012; Edmondson 2011). To address this challenge, organizations need to (a) make valuable help as easily accessible as possible (Bamberger 2009; Frese and Keith 2015), and (b) lower the perceived social risk of communicating about errors (Bamberger 2009; Edmondson 2011; Hofmann et al. 2009). Both goals can be addressed through the concept of the organization's learning from error climate.

Putz et al. (2012) definition of learning from error climate as stated in the introduction, as well as Jenkins et al.'s (2008, p. 47) work states that climate "establishes the parameters of acceptable and unacceptable behavior". Putz et al. (2012) developed the concept of an organization's learning from error climate based on an extensive literature review and include the behaviors of the direct supervisor, behavior of colleagues, the way work is structured and organized, and the values shared by members of an organization in the concept. In the authors' view, a supportive learning climate makes it possible for individuals to observe their colleagues engaging in learning from errors themselves, communicating the desirability of learning behaviors after making an error. This does not imply a purely optimistic view of errors, especially not when the same errors are made repeatedly; the concept rather focuses on the conditions necessary for realizing the positive outcome of social learning after an error occurs, and not on making errors as an indication of poor ability (Putz et al. 2012). An organization's learning from error climate therefore captures individuals' perceptions of the work environment at the organizational level. ${ }^{2}$

Studies across several professions have explored the relationship between an organization's learning from error climate as well as related related concepts and

\footnotetext{
${ }^{2}$ As such, this concept is distinct from other concepts such as an organization's error (management) climate / culture (Gold et al. 2014; van Dyck et al. 2005) and from the concept of negative knowledge (e.g. Bauer et al. 2012). An error management climate / culture acknowledges that errors are not perfectly preventable and emphasizes the creation of structures for managing the negative (e.g. financial) or positive (e.g. learning) consequences of errors (van Dyck et al. 2005). In line with the focus of the present study, the learning from error climate specifically focuses on mechanisms that enable the positive error outcome of learning (Putz et al. 2012). The theory of negative knowledge on the other hand describes an important outcome of learning from errors - what not to do (Bauer et al. 2012). The mechanisms and conditions under which this knowledge is developed however is not consistently described; consequently, we rely on the specific 'learning from error climate' to address the present research question.
} 
individuals' behaviors after making an error provides a clear and positive picture. Studies by Perreault et al. (2012) and Gold et al. (2014) demonstrate that a supportive error climate (a related and broader concept) is associated with an increased likelihood of reporting errors to colleagues, and especially to hierarchical superiors. Focusing more specifically on learning from errors, substantial work has been done on the influence of organizations' climate on individuals' learning from error. Evidence from healthcare (Edmondson 2004; Tucker and Edmondson 2003) and aviation (Helmreich 2000) shows a positive relationship between a firm or unit's climate and long-term learning from errors. Carmeli and Gittell (2009) find that when professionals - across a range of domains - felt they could talk about errors without fearing negative consequences, they showed more learning from errors behavior. At the organizational level, we therefore expect a supportive learning from error climate to be associated with more frequent help-seeking, and to higher learning value of help sought:

H2a: Young professionals who perceive a more supportive learning from error climate will seek help more frequently.

$\mathrm{H} 2 \mathrm{~b}$ : Young professionals who perceive a more supportive learning from error climate will seek help of higher learning value.

However, in these existing studies, learning from error climate has not been explicitly related to the two barriers to help-seeking: accessibility and relative hierarchy. Not only do we expect that an organization's learning from error climate will relate directly to individuals' frequency of help-seeking and learning value of help sought, but we also expect it to moderate the relationship between relative hierarchy and learning value of help sought. In a supportive learning environment, social costs of help-seeking should be lower than in an unsupportive climate, which would enable individuals to seek help from more valuable sources, independent from accessibility. Based on the findings reported above, a supportive learning from error climate is expected to positively moderate the relationship between relative hierarchy and the learning value of help sought, leading to our final hypotheses:

H3a: Young professionals who perceive a more supportive learning climate will seek help more frequently from hierarchical superiors.

$\mathrm{H} 3 \mathrm{~b}$ : Young professionals who perceive a more supportive learning climate will seek help of higher learning value from hierarchical superiors.

\section{Methods}

\section{Setting, Sample and Procedure}

The current study was carried out in the professional setting of auditing. Auditors assess financial statements of organizations and provide assurance that the financial statements follow the applicable accounting laws and regulations. This work is carried out in a hierarchical team setting: young professionals (juniors) collect and explore evidence through mostly standardized tasks and structures. In this work, so-called seniors are in charge of supervising the juniors. These seniors have a few years of work experience 
with the same structured tasks, mostly within the same audit firm. Seniors are in charge of coaching the juniors and of providing help when needed. Above the senior level, a manager is in charge of reviewing the work done at lower levels and who can be consulted for complex questions where necessary (Abdolmohammadi 1999; Owhoso et al. 2002). Due to this fixed hierarchical structure, the audit setting differs e.g. from medical or educational contexts; supervisors of young professionals are acutely aware of the tasks performed and part of their job description is the coaching of juniors.

The audit profession is well-aware of the role that errors play in their work: errors made by juniors may escalate through the hierarchical levels, and if not corrected, can threaten the quality of audits delivered, along with the reputation of the audit firm (Gold et al. 2014; Gronewold and Donle 2011; Stefaniak and Robertson 2010). The hierarchical review structure and the coaching system are designed to facilitate the detection and reporting of errors, including the provision of help. In their interview study, Westermann et al. (2015) found that auditors perceive this system to be helpful in providing access to knowledgeable colleagues, but at the same time, this study also showed that working together with different teams and sometimes only for a short time can increase the barriers of timely asking for help, depending on how the teamwork is managed. This makes auditing a relevant setting for exploring the seeking of help as a means of learning after making an error: the underlying work structure is supportive of help-seeking, but social barriers exist equally (focus on managing errors, emphasis of audit quality, competitive business structures).

Data for this study were collected during mandatory training sessions attended by Dutch junior auditors as part of their audit certification. These sessions are run by universities and participants work at a variety of firms. The design of the data collection procedure focused on assuring anonymity and addressing desirability bias in five principal ways. First, data were collected in the presence of an unfamiliar researcher; the trainers were unaware of the content of the study and left the room before the briefing. The researcher's work lies outside the audit domain and he/she exclusively monitored the process, providing technical help where necessary. Second, all data were collected using an online questionnaire, in which identifying information was kept to a minimum (months of work experience, gender, and firm type). Third, the network prompts emphasized the use of initials or first names, not actual names, to assure participants of our interest in tie characteristics, rather than identifying the ties themselves. Fourth, participants could not self-select into participation, since complete cohorts of training attendants completed the instruments. Finally, the questionnaire was built on previously validated scales, including two separately developed measures for how individuals perceive the organizational value placed on learning from errors. Information from the different measures was then compared to data from the respective validation papers to ensure validity.

In total, 173 participants completed the online questionnaire. Sixteen participants were excluded prior to analysis because they had worked at their firm less than one month, which did not allow them to fill out the survey items reliably. The working sample for this study therefore comprises 157 junior auditors. Work experience ranges from one to 36 months, with an average of 10.31 months. Participants worked for eleven different audit firms, with $86.11 \%$ of participants working for one of the Big 4 firms; $64 \%$ of the participants are male. 


\section{Measures for Frequency of Help-Seeking and Learning Value of Help Sought}

The dependent variables in this study, frequency and learning value of help sought after errors, are operationalized following social network analysis methodology. Social network analysis (Borgatti and Cross 2003) is used to measure auditors' help-seeking behavior, building further on van der Rijt et al. (2013). Social network analysis is an established tool to investigate interactions between individuals with a specific purpose (Hatala 2006; Hatala and Fleming 2007; Scott 2013). We follow van der Rijt et al.'s (2013) methodology for capturing individuals' ego-networks (Cross and Sproull 2004). This specific approach was chosen because it is an established method for researching social processes while being unable to observe participants directly and without being able to question participants after the occurrence of specific experiences.

To address our research question whether an organization's learning from error climate enables young professionals to overcome the barriers of accessibility and hierarchical boundaries, to enable help-seeking after making an error, we formulated the following prompt for participants to generate relevant ties: "when you experience an error or mistake at work, who do you typically turn to for help and advice? Please indicate the initials or first names of the most relevant persons". This prompt was chosen to focus on the undertaking of social learning from error behavior, rather than on individual learning behavior, in line with our theoretical framework. We chose to include the term error as well as mistake in the prompt, as they are frequently used interchangeably in practice (Edmondson 2012) and would be used more or less frequently across different firms. Subsequently, we provided ten slots for participants to indicate ties, the average of Wolfe Morrison (2012) maximum number of ties reported in her pilot interviews, and the observed maximum of ties as indicated in the research by van der Rijt et al. (2013). For each subsequent question, the ties named in response to the prompt are reproduced, and participants evaluate the characteristics of each tie in turn.

For the dependent variables, participants indicated for each tie how often they seek help from this person, ranging from (almost) never to very often on a scale from 1 to 6 , and in how far the help they received from this tie contributed to their learning, ranging from 'does not contribute at all' to 'contributes a lot', on a scale from 1 to 5 .

\section{Measures for Accessibility, Relative Hierarchy, and Learning from Error Climate}

Help-seeking network characteristics were measured with two additional network questions adapted from van der Rijt et al. (2013). Accessibility of ties is measured by asking participants to indicate how often they worked together with each tie outside of seeking help, ranging from (almost) never to very often, a scale from 1 to 4 . For the variable relative hierarchy, participants indicated for each tie whether they worked at a lower, the same, a higher hierarchical level, or outside of the firm, resulting in a categorical variable with four values to operationalize relative hierarchy.

Given the sensitive nature of error-related topics, we also took specific measures for the second part of this study's measurement. We measure participants' perceptions of the value attached to learning from errors at their firms through two independently developed and validated measures. First, we used the short version of Putz et al.'s (2012) organizational learning from error (OLE) questionnaire. The 16-item scale 
contains items such as "employees in our work group know how to get the information they need to correct errors" and "when someone in our work group makes a mistake, other co-workers will help him/her to fix it", rated from 1 to 6 . The scale has a high reliability with a Cronbach alpha of 0.886 , and descriptives do not deviate significantly from the validation sample's in Putz et al. (2012). Next to the OLE questionnaire, we employed a second well-established measure, Rybowiak et al.'s (1999) error orientation questionnaire. This questionnaire measures individuals' predispositions to reacting to errors. Rybowiak et al. (1999) state that these predispositions are developed over time based on error experiences in a specific environment. We have selected two subscales of Rybowiak et al.'s (1999) questionnaire that we expect to relate to our measures of help-seeking: covering up errors and learning from errors. Both scales are measures through 4-item Likert-type scales ranging from 1 to 5 . The covering up errors scale contains items such as "why mention a mistake when it is not obvious?" and "it is disadvantageous to make one's mistakes public", and has a Cronbach alpha of 0.669. The learning from errors scale contains items such as "mistakes assist me to improve my work" and "mistakes provide useful information for me to carry out my work", with a Cronbach alpha of 0.853 .

\section{Analysis}

The data collected for this study are nested: characteristics and perceptions of the individual auditor are expected to influence the nature of help-seeking behavior with different ties. To account for this nested data structure, multilevel modelling is used to discern the relative influence of variables across these two levels, as well as the relative amount of variance explained (van Duijnen et al. 1999). In our analysis of the first two hypotheses, we follow the analysis strategy suggested by Heck et al. (2014). In a first step, a null model is calculated to understand variance at the two levels of analysis when only taking into account the dependent variable. Second, independent variables at the different levels are added separately to the null model to understand their relative contribution to explaining both types of variables (model 1-3). In a final step, all variables are included at the same time to understand their simultaneous effect (model 4). To evaluate the effectiveness in explaining variance, models calculated in steps 2 and 3 are compared to the null model. Before the analysis, all individual level variables were grand mean centred and scores of participants from different firms on these different variables were explored using ANOVA. We find small differences with respect to the network characteristics as well as learning from error climate. Because these differences showed low effect sizes ranging from $\omega^{2}=0.027$ and $\omega^{2}=0.047$, we chose not to include firm membership as another level in the analysis. To address the third hypothesis, we employ two analyses. ANOVA is used to compare the helpseeking frequency and learning value of help sought from hierarchical superiors depending on the participants' perceived learning from error climate. Scores for learning from error climate, covering up errors and learning from errors are mediansplit into below- and above-median groups, and $\omega^{2}$ is used as an effect size measure. As a follow-up, we use single-level moderation analysis, using mean values of the dependent variable learning value of help sought and the independent variable of relative hierarchy, to further investigate hypothesis $3 \mathrm{~b}$. In the moderation analysis, we follow Hayes' (2013) procedure for calculation and visualization of interaction effects. 


\section{Results}

\section{Descriptive Results}

In total, this survey covers 718 ties. On average, participants reported to contact 4.484 ties after experiencing an error $(\mathrm{SD}=2.493)$, asking for help either several times a week or daily $(\mathrm{M}=3.582, \mathrm{SD}=1.062)$, and that the help obtained provides a positive value for learning $(M=4.143, S D=0.805)$. The learning from error climate was reported to be mostly supportive $(M=4.488, S D=0.622)$, and on average, help was sought from colleagues of the same hierarchical level, with a slight upward orientation $(\mathrm{M}=2.681$, $\mathrm{SD}=0.718$ ), and from colleagues that participants work with 'sometimes' to 'frequently' $(\mathrm{M}=2.724, \mathrm{SD}=0.682)$. Finally, participants provided a low disposition to cover up errors $(\mathrm{M}=1.910, \mathrm{SD}=0.644)$ and a high disposition to learning from errors $(\mathrm{M}=$ 4.191, $\mathrm{SD}=0.523)$.

Based on Nadler et al.'s (2003) finding that frequency of help-seeking may relate non-linearly to performance, we explore the relationship between our two dependent variables, frequency of help-seeking and learning value of help sought. Figure 1 illustrates a concave curve showing that a higher help-seeking frequency is related to decreasing marginal returns in terms of learning value of help sought. This finding provides a context in which to interpret the results of our first hypothesis.

\section{Hypothesis 1: Frequency of Help-Seeking after Errors}

To investigate whether help-seeking frequency is explained by the tie-level predictors of hierarchy and accessibility (hypothesis 1a), by the individual-level predictors of error orientation (hypothesis 2a) and perceived learning from error climate (hypothesis 3a), we developed a multilevel model. Table 1 reports the results for the first part of our three hypotheses. The null model estimates variance at the two levels of analysis, tie level and individual level, providing a baseline for evaluating models 1 to 4 . The interclass correlation (ICC) of 0.385 indicates that variance between individual auditors is larger than between ties, warranting the multilevel approach.

Model 1 adds the variables at the tie level: relative hierarchy and accessibility. Participants indicated to seek help more frequently when they could contact colleagues at the same hierarchical level $(\hat{y}=0.570$, S.E. $=0.244)$, and when they could contact colleagues whom they work with frequently $(\hat{y}=0.720$, S.E. $=0.041)$. Model 1 is a significant improvement over the null model, as indicated by the comparison of the -2 log likelihoods $\left(\mathrm{X}^{2}(2)=301.298, p<0.000\right)$. Using Heck et al.'s $(2014)$ approximation of variance explained [(level 1 variance $_{\text {null }}$ - level 1 variance $\left._{1}\right) /$ level 1 variance $\left._{\text {null }}\right)$ ], we find that tie-level predictors explain an addition $24.2 \%$ of variance. These findings provide support for hypothesis 1a.

Model 2 explores the influence of individual-level predispositions towards covering up errors and learning from errors. Only the predisposition to cover up errors significantly (and negatively) explains the frequency of help-seeking $(\hat{y}=-0.351$, S.E. $=$ 0.120 ), also presenting a significant improvement over the null model, if to a smaller degree than model $1\left(\mathrm{X}^{2}(2)=9.653, \mathrm{p}<0.000\right.$, change in proxy for $\left.\mathrm{R}^{2}=0.098\right)$. This provides partial support for hypothesis $2 \mathrm{a}$. 


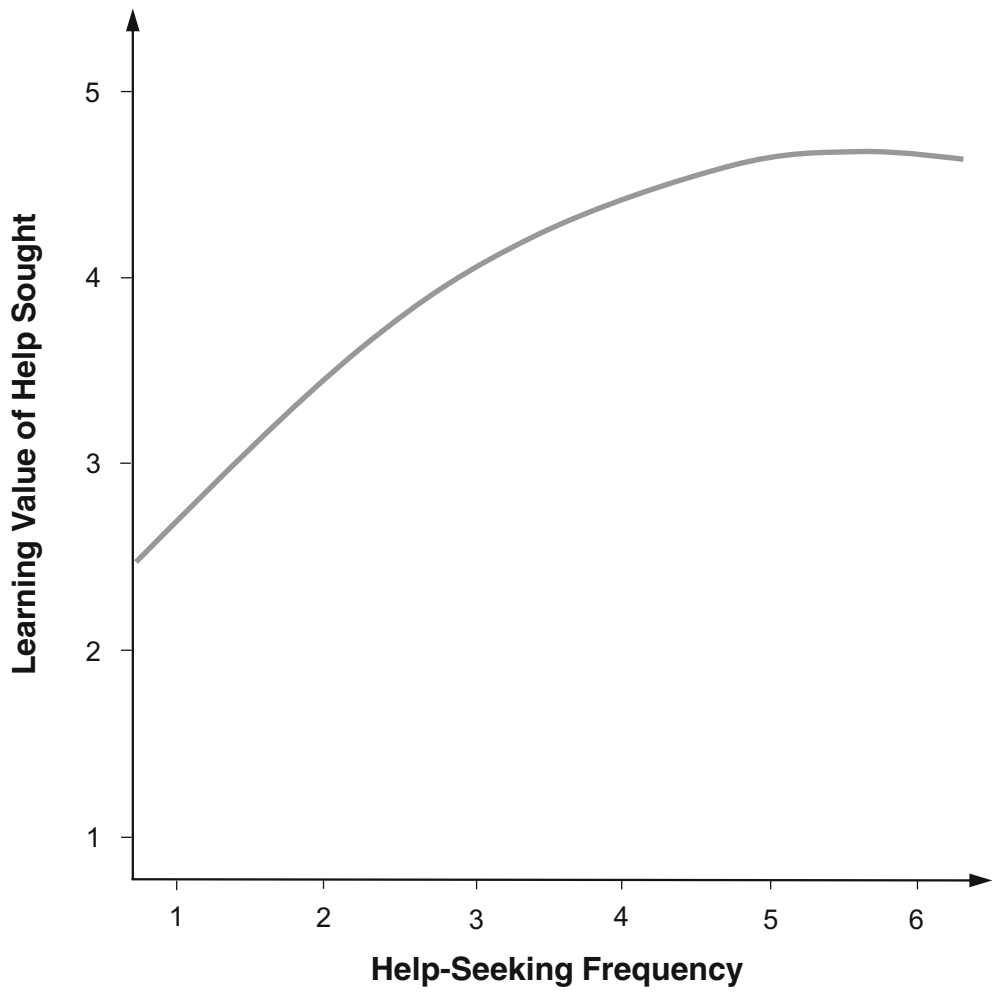

Fig. 1 The non-linear relationship between help-seeking frequency and the learning value of help sought; $\mathrm{R}^{2}=0.267$

Model 3 in turn uses a firm's perceived learning from error climate as a predictor of help-seeking frequency. While the estimated coefficient is not significant $(\hat{y}=0.247$, S.E. $=0.315)$, the model is a significant improvement over the null model, again to a lesser degree than model $1\left(\mathrm{X}^{2}(1)=5.687, p<0.025\right.$, change in proxy for $\left.\mathrm{R}^{2}=0.061\right)$. This finding represents weak support for hypothesis $3 \mathrm{a}$.

In a final step, we estimate the complete model, which is a significant improvement over the null model $\left(\mathrm{X}^{2}(6)=305.130, \mathrm{p}<0.000\right)$, explaining an additional $27.4 \%$ of variance in the frequency of help-seeking. As expected based on models 1 to 3, tie-level predictors crown out effects at the individual level. Auditors seek help frequently when they can contact their immediate colleagues who are employed at the same hierarchical level. Individual predispositions and the firm's learning from error climate play a minor role in encouraging frequent help-seeking.

\section{Hypothesis 2: Learning Value of Help Sought after Errors}

The same analysis as presented above is carried out to explore in how far the learning value from help sought is explained by tie-level factors (hypothesis 1b), individual predispositions (hypothesis 2b) and the firm's learning from error climate (hypothesis $3 b$ ). Results are reported in Table 2. The null model, the baseline, reveals that significant variance exists both between individuals and between ties, resulting an ICC of 0.396 . 


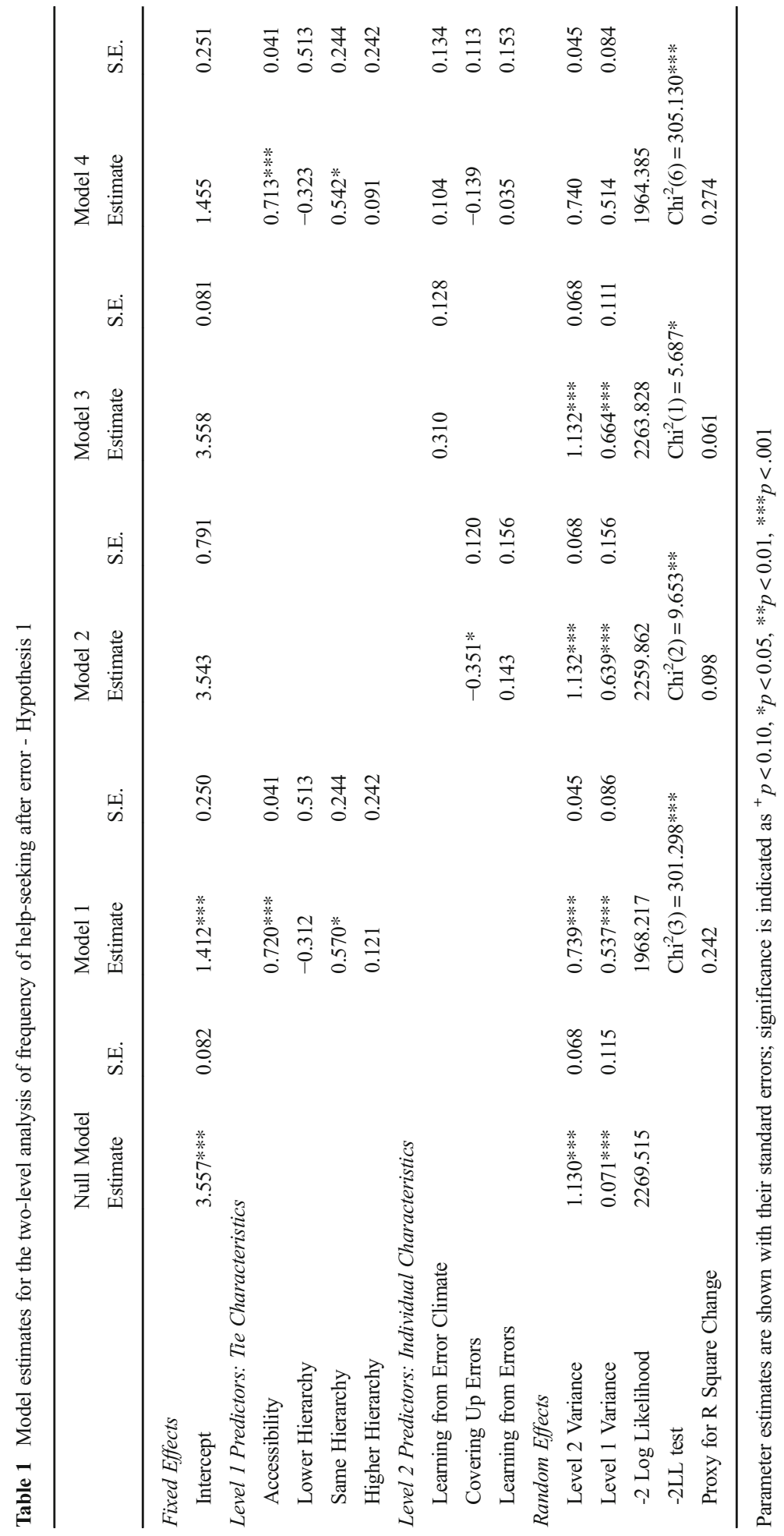




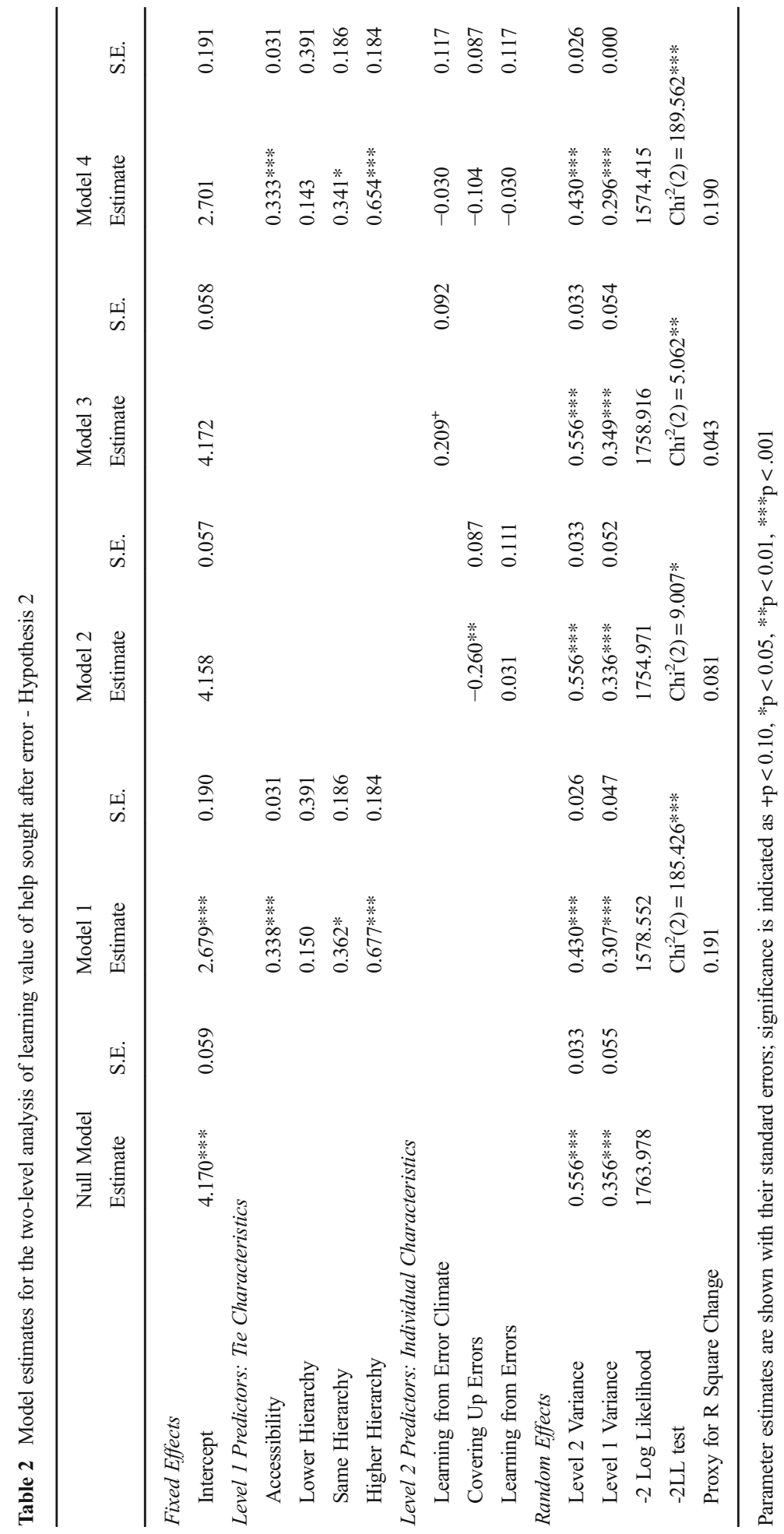


Model 1 explores hypothesis $1 \mathrm{~b}$, looking at tie-level predictors of relative hierarchy and accessibility. Help is perceived to be most valuable for learning when sought from colleagues at the same hierarchical level $(\hat{y}=0.365$, S.E. $=0.186)$, from colleagues at higher hierarchical levels $(\hat{y}=0.677$, S.E. $=0.184)$, as well as from easily accessible colleagues $(\hat{y}=0.336$, S.E. $=0.031)$. With an additional $19.1 \%$ of variance explained, Model 1 represents a significant improvement over Model $2\left(\mathrm{X}^{2}(2)=185.426, \mathrm{p}<0.000\right)$. This provides us with strong support for hypothesis $1 \mathrm{~b}$.

Model 2 and 3 show the relationships between individual-level factors on learning value of help received. Model 2 shows that the predisposition of covering up errors negatively explains learning value $(\hat{y}=-0.260$, S.E. $=0.087)$, and Model 3 shows a positive relationship for learning from error climate $(\hat{y}=0.209$, S.E. $=0.092)$. Both models 1 and 2 are improvements over the null model, but explain less variance than model $1\left(\mathrm{X}_{2}{ }_{2}(2)=9.007, \mathrm{p}<0.025 ; \mathrm{X}_{3}{ }_{3}(1)=5.062, p<0.010\right)$. These findings provide support for hypotheses $2 \mathrm{~b}$ and $3 \mathrm{~b}$.

Model 4, the overall model, reveals a similar pattern, explaining help-seeking frequency. The improvement over the null model is significant $\left(\mathrm{X}^{2}(6)=189.562, \mathrm{p}<\right.$ 0.000 , variance explained 19\%). Again, effects at the tie level crowd out effects at the individual level. Auditors seek valuable help from easily accessible colleagues, especially from higher hierarchical level, and to a lesser degree also from colleagues of the same level.

\section{Hypothesis 3: Interaction between Hierarchy and Climate Measures}

To investigate the third hypothesis, we first explore the difference in help-seeking frequency (hypothesis 3a) and learning value of help sought (hypothesis 3b) from hierarchical superiors between participants who perceive an above- or below-median learning from error climate. For comparison, we have also included the differences for hierarchical equals. The results of the ANOVA are reported in Table 3. Regarding hypothesis 3a, we explore differences in help seeking frequency from hierarchical superiors and equals given learning from error climate. First, we found that participants who perceive a more supportive organizational learning from error climate seek help significantly more frequently than their peers in a less supportive climate $(\mathrm{F}=12.963$, $p=0.000 ; \omega^{2}=0.079$ ). However, we did not find a significant difference with respect to seeking help from hierarchical superiors. Next, focusing on covering up errors, we found that participants who are less likely to cover up errs sought help more frequently from hierarchical superiors $\left(\mathrm{F}=9.933, p=0.002, \omega^{2}=0.018\right)$ and from hierarchical equals $\left(\mathrm{F}=3.492, p=0.064, \omega^{2}=0.017\right)$. We did not find significant differences for learning from errors, lading us to conclude that this study provides tentative support for hypothesis 3a. For hypothesis $3 \mathrm{~b}$, we did not find differences in learning value of help sought from hierarchical superiors or equals for organization learning from error climate or learning from errors. However, participants who scored low on covering up errors sought help of higher learning value from hierarchical superiors $\left(\mathrm{F}=3.876, p=0.050, \omega^{2}=0.005\right)$, providing equally tentative support for hypothesis $3 b$.

To follow up on hypothesis $3 b$, three multiple regressions were calculated with interactions between the mean value of relative hierarchy and learning from error 


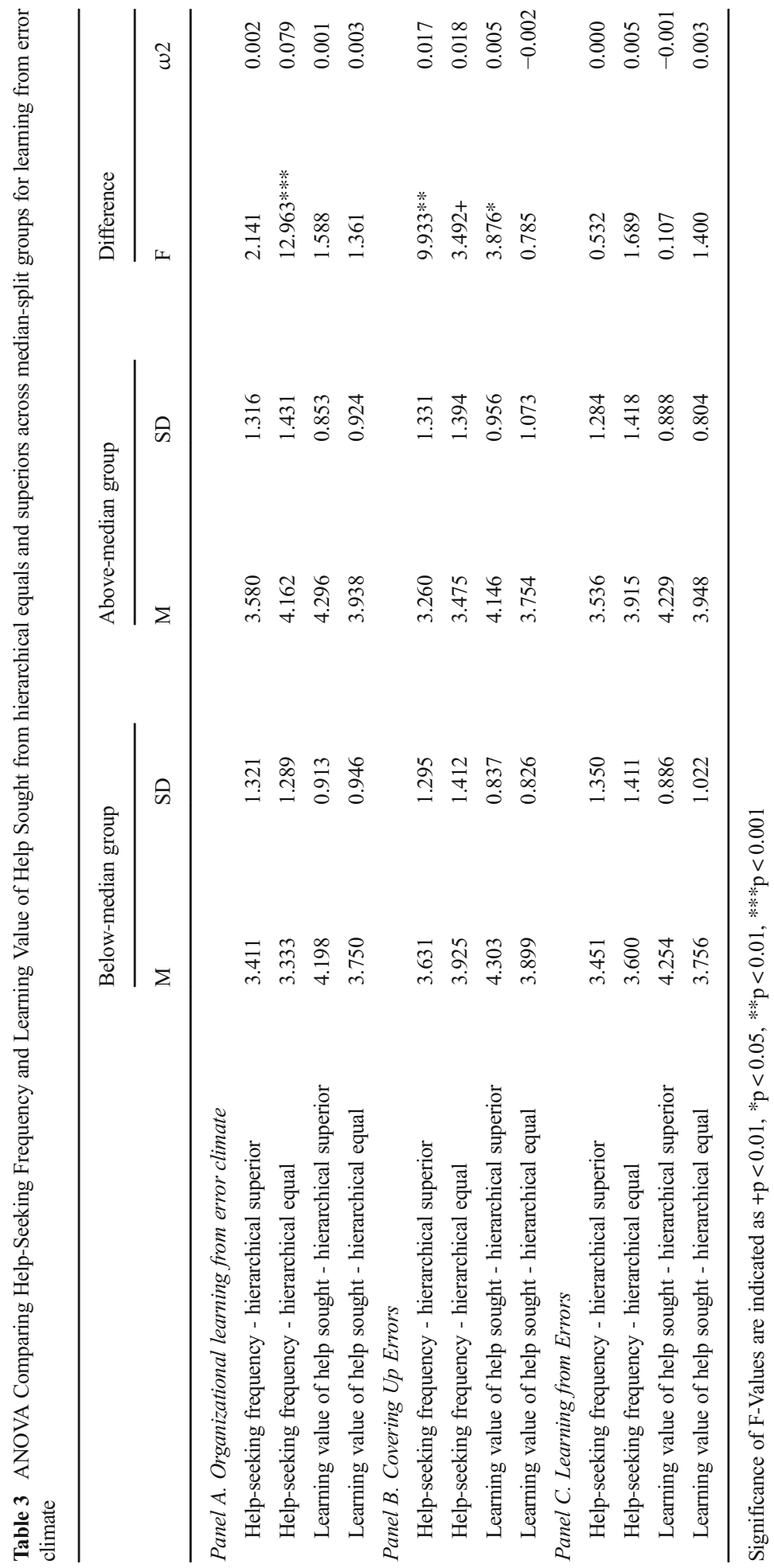




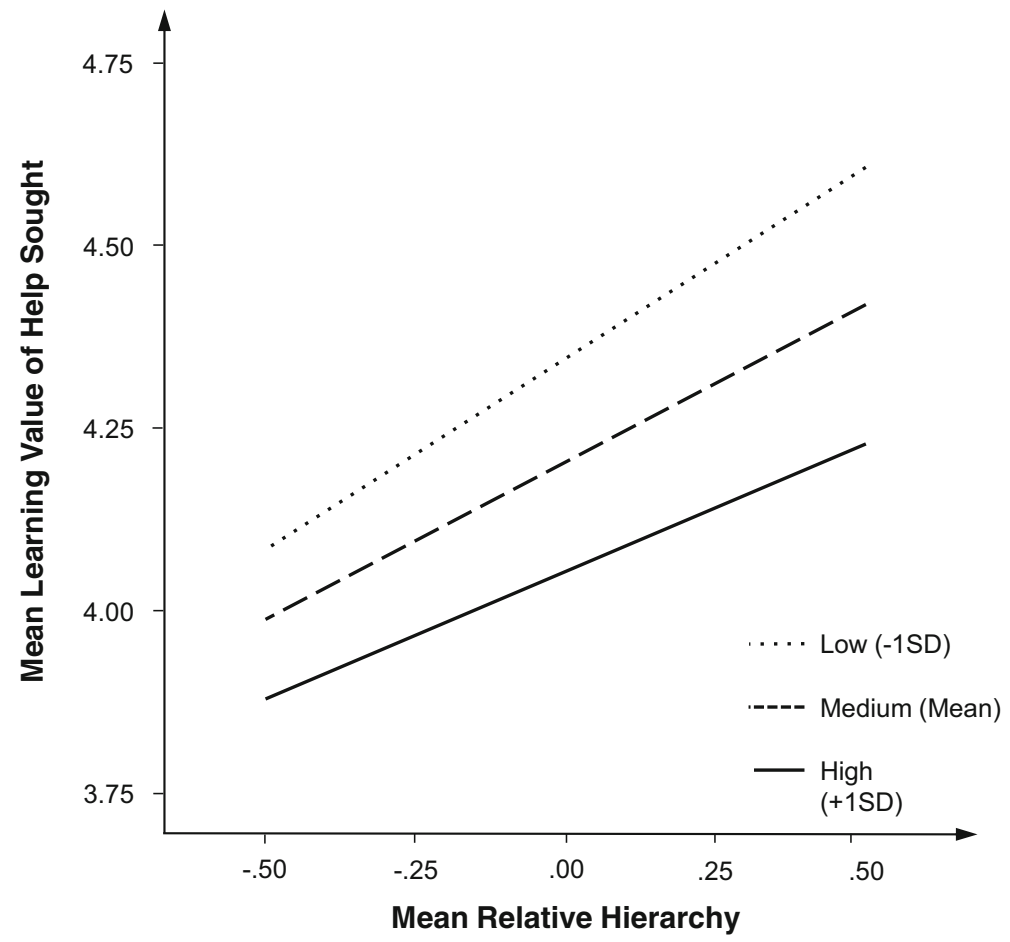

Fig. 2 A visual representation of the interaction between tie hierarchy and covering up errors, at three values of covering up errors $(-1 \mathrm{SD}$, mean, $+1 \mathrm{SD})$; the overall interaction is not significant $(p=.508)$; each of the conditional effects is significant at $p<0.050$

climate (Model 1), covering up errors (Model 2), and learning from errors (Model 3). Using Hayes' (2013) PROCESS macros for SPSS, estimates for interactions are derived, and the interaction effects are visualized by investigating the relationship between relative hierarchy and learning value of help sought for three values of the moderators: the mean minus one standard deviation (the low group), the mean value (the medium group), and the mean plus one standard deviation (the high group) (Figs. 2, 3 and 4). With respect to learning from error climate, we found a marginally significant interaction $(\beta=0.492, p<0.10)$, in the expected direction. The more supportive participants perceive their organizational learning from error climate to be, the more they value help received from their superiors, starting at the medium group $(\beta=$ $0.553, p<0.001)$ and peaking in the high group $(\beta=0.861, \mathrm{p}<0.001)$. This interaction is visualized in Fig. 4, supporting hypothesis $3 \mathrm{~b}$. Next, we find that covering up errors does not significantly moderate the relationship between relative hierarchy and learning value from help sought ( $\beta=-.0162, p>0.01$ ). The conditional effects however demonstrate that the positive relationship between these two variables is only significant for participants in the low and medium groups $(\beta=0.633, p<0.01$; and $\beta=0.528, p<$ 0.001 , respectively). This interaction is visualized in Fig. 2 , and the findings provide mixed support for hypothesis $3 \mathrm{~b}$. Finally, the interaction between relative hierarchy and learning from errors is significant $(\beta=-0.597, p<0.001)$, but the direction of the effect is contrary to our hypothesis. Participants who report a high predisposition towards learning from error do not perceive help to be more valuable from hierarchically 


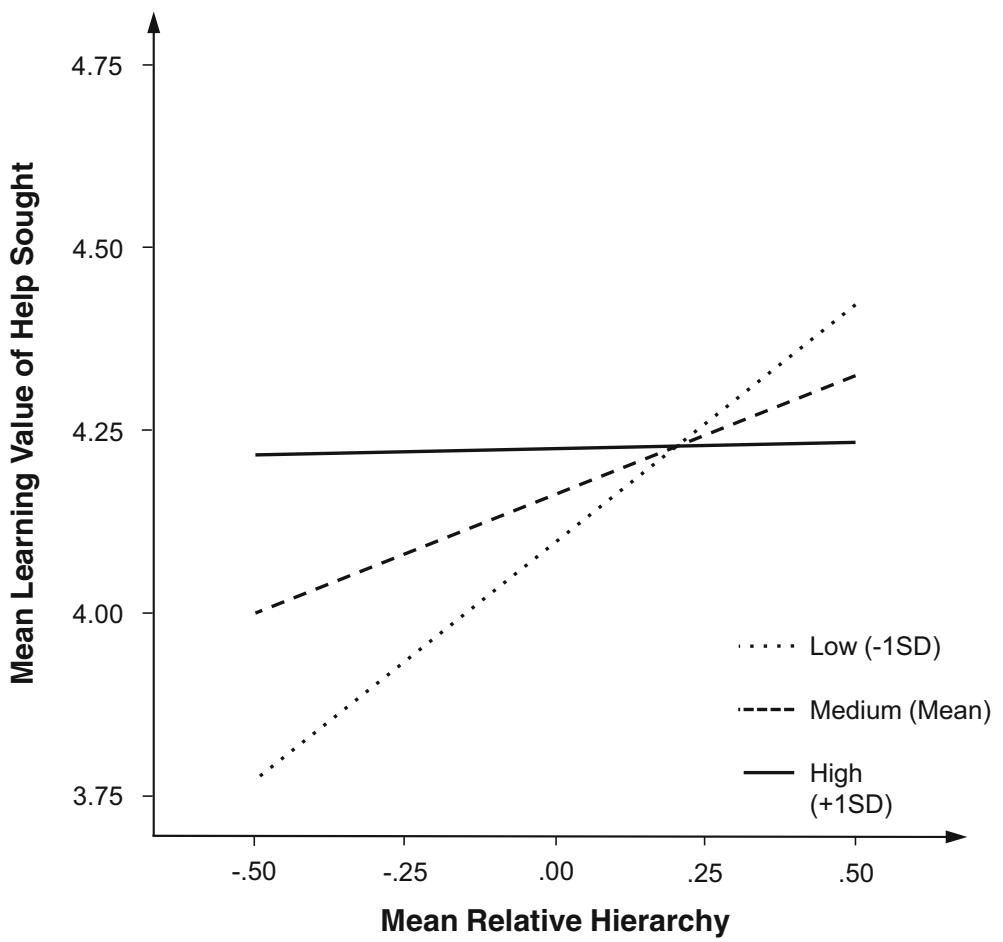

Fig. 3 A visual representation of the interaction between tie hierarchy and learning from errors, at three values of learning from errors $(-1 \mathrm{SD}$, mean, $+1 \mathrm{SD})$; the overall interaction is significant $(p=.001)$; the low $(\mathrm{p}=.000)$ and medium $(p=.013)$ conditional effects are significant at $p<0.050$

superior colleagues $(\beta=0.017, \mathrm{p}>0.01)$. Instead, those participants in the low and medium groups value help received more when it comes from higher hierarchy levels ( $\beta=0.655, \mathrm{p}<0.001$; and $\beta=0.336, p<0.05$, respectively). This interaction is visualized in Fig. 3. This result is in contrast to our hypothesis $3 b$ (Table 4).

\section{Discussion}

In this study we explored two specific learning from errors behaviors: (1) the frequency with which young professionals seek help after making an error themselves, and (2) the learning value of the help they sought within their social network (Bamberger 2009; Borgatti and Cross 2003; Van Der Rijt and Van Den Bossche 2013). For each, we investigated the influence of two common barriers to help-seeking: accessibility to help sources (Borgatti and Cross 2003; van der Rijt et al. 2013) and emotional and social costs, as embodied by relative hierarchy (Bamberger 2009; Hofmann et al. 2009). Moreover, we explored whether an organization's learning from error climate (Edmondson 2011; Putz et al. 2012; Rybowiak et al. 1999) helps individuals to overcome these two barriers, increasing learning from errors. Findings for each of the two learning from error behaviors are discussed in turn, followed by a discussion of limitations and implications for future research as well as for practice. 


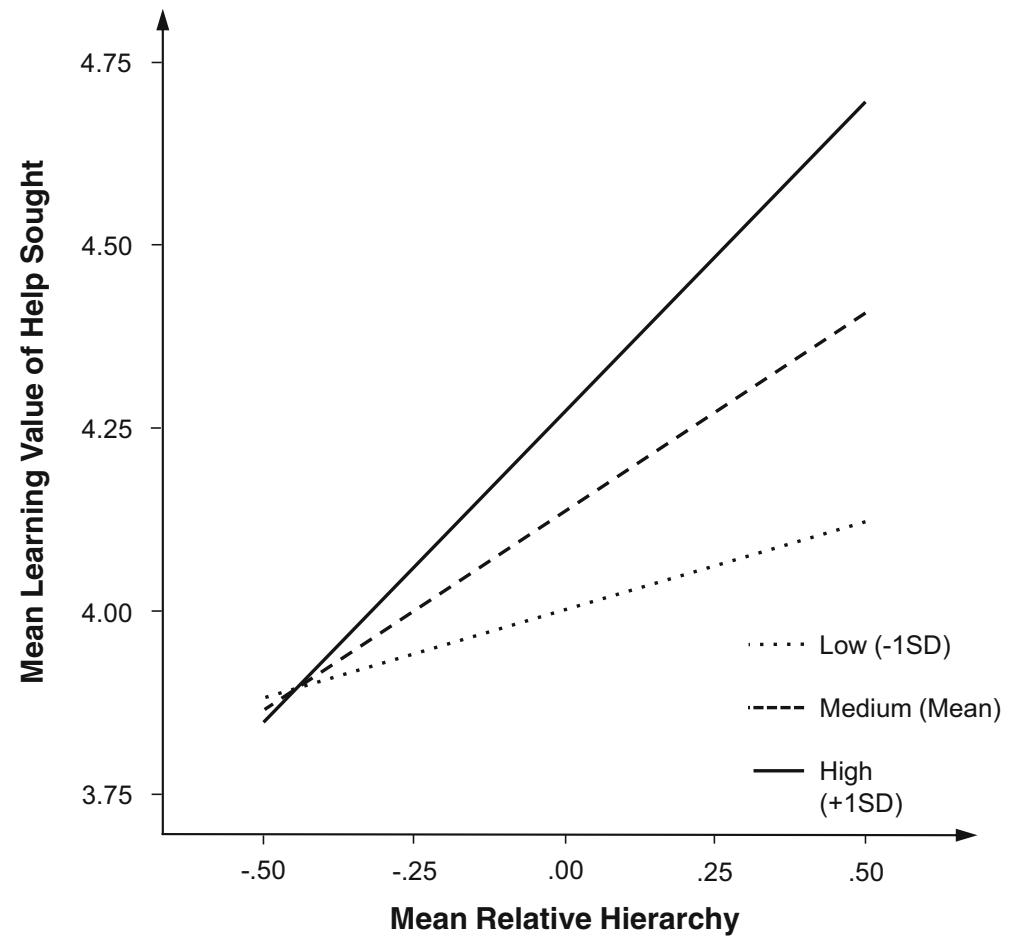

Fig. 4 A visual representation of the interaction between tie hierarchy and learning from error climate, at three values of learning from error climate $(-1 \mathrm{SD}$, mean, $+1 \mathrm{SD})$; the overall interaction is significant $(p=.062)$; the medium $(p=.000)$ and high $(\mathrm{p}=.000)$ conditional effects are significant at $p<0.000$

\section{Increasing the Frequency of Help-Seeking after Making an Error}

To begin our analysis, we were interested in understanding the drivers behind young professionals' frequency of help-seeking. We observed that help-seeking frequency results in marginally decreasing returns on learning value of help sought, in line with Nadler et al. (2003). The multilevel analysis of the two network barriers to help-seeking and learning from error climate revealed that most variance is explained at the level of the network barriers: young professionals in this study sought help most frequently from those ties that are highly accessible, especially those at the same hierarchical level. This is in line with findings by Bamberger (2009), Hofmann et al. (2009) and Tucker and Edmondson (2003). Both Bamberger (2009) and Hofmann et al. (2009) observed that seeking help frequently from 'accessible friends' may be indicative of help-seeking not for the purpose of learning, but for the purpose of reassurance. Tucker and Edmondson (2003) formulated their observation in the healthcare sector more sharply: seeking reassurance from similar connections was found to be indicative of high social costs to talking about errors, and was found to relate to either an unsupportive or an inconsistent climate at the nurses' units. Next, we find a limited role of individuals' beliefs with respect to covering up and learning from errors (Rybowiak et al. 1999) and perceptions of the organization's learning from error climate (Putz et al. 2012) in helpseeking frequency. Only individual beliefs with respect to covering up errors are negatively related to frequency of help-seeking, as found earlier in the healthcare 
Table 4 Moderation analysis of climate measures on the relationship between relative hierarchy and learning value of help sought after error - Hypothesis $3 b$

\begin{tabular}{|c|c|c|c|c|c|c|}
\hline & Model 1 & & Model 2 & & Model 3 & \\
\hline & Estimate & S.E. & Estimate & S.E. & Estimate & S.E. \\
\hline Constant & $4.138 * * *$ & 0.062 & $4.143 * * *$ & 0.062 & $4.160 * * *$ & 0.061 \\
\hline \multicolumn{7}{|l|}{ Direct Relationships } \\
\hline Relative Hierarchy & $0.553 * * *$ & 0.133 & $0.528 * * *$ & 0.134 & $0.336^{*}$ & 0.134 \\
\hline Covering Up Errors & & & $-0.273 * *$ & 0.098 & & \\
\hline Learning from Errors & & & & & 0.123 & 0.116 \\
\hline Learning from Error Climate & $0.220 *$ & 0.1 & & & & \\
\hline \multicolumn{7}{|l|}{ Interactions } \\
\hline Hierarchy $\times$ Covering Up Errors & & & -0.162 & 0.244 & & \\
\hline Hierarchy $\times$ Learning from Errors & & & & & $-0.597 * * *$ & 0.176 \\
\hline $\begin{array}{l}\text { Hierarchy } \times \text { Learning from Error } \\
\text { Climate }\end{array}$ & $0.492+$ & 0.261 & & & & \\
\hline \multicolumn{7}{|l|}{ Conditional Effects } \\
\hline Low (-1SD) & 0.245 & 0.183 & $0.633 * *$ & 0.235 & $0.655 * * *$ & 0.137 \\
\hline Medium (mean) & $0.553 * * *$ & 0.133 & $0.528 * * *$ & 0.134 & $0.336^{*}$ & 0.134 \\
\hline High (+1SD) & $0.861 * * *$ & 0.861 & 0.423 & 0.175 & 0.017 & 0.187 \\
\hline \multicolumn{7}{|l|}{ Model Information } \\
\hline$F$ Value & & $7.752 * * *$ & & $8.211 * * *$ & & $9.643 * * *$ \\
\hline R Square & & 0.14 & & 0.147 & & 0.168 \\
\hline R Square Change from Interactions & & $0.021+$ & & 0.003 & & $0.067 * * *$ \\
\hline
\end{tabular}

Parameter estimates are shown with their standard errors; significance is indicated as $+\mathrm{p}<0.10, * \mathrm{p}<0.05$, **p $<0.01, * * * \mathrm{p}<.001$

setting by Bauer and Mulder (2013) and (Leicher et al. 2013). The observation of the crowding out of these factors may relate to what Cha and Edmondson (2006) refer to as 'value hypocrisy': stating that learning is valued within an organization does not automatically result in behaviors in line with these professed values. This may also be the case here: even if young professionals perceive a supportive learning from error climate, this may not automatically translate into more frequent help-seeking in direct interactions with others. To conclude, we find that young professionals in this setting sought frequent help from members of their network that are first and foremost accessible, mostly at the same hierarchy level, while learning from error climate and individual error-related beliefs only played a minor role.

\section{Increasing the Learning Value of Help Sought after Making an Error}

Similarly to the findings regarding frequency of help-seeking, the learning value of help sought relates very strongly to the two network barriers to help-seeking, with a smaller influence of learning from error climate and error-related beliefs. Contrary to the frequency of help-seeking however, it is relative hierarchy over accessibility that determines learning value of help sought. Young professionals report to receive help of high learning value from hierarchical superiors more than from colleagues on the 
same level; this finding is the opposite of the results for frequency of help-seeking. This finding remains constant also when comparing participants' help-seeking behavior in supportive and unsupportive learning from error climates. Accessibility plays a significant role in learning value of help sought, but explains less variance than relative hierarchy. This finding mirrors earlier results reviewed by Bamberger (2009) and Frese and Keith (2015) - hierarchical superiors provide more valuable help, but are at the same time less frequently asked for help, reflecting the valuing of knowledge in the presence of higher social and emotional costs. Addressing the relationship between relative hierarchy and learning value of help sought, we found that those participants scoring low on covering up errors sought help of higher learning value both from hierarchical superiors and from hierarchical equals. This finding aligns with our hypothesis based on Bamberger's (2009) view that social costs play a role in obtaining valuable help: learning from error-related beliefs and climate appear to lower social and emotional costs, making the seeking of valuable help more likely. The observation that when covering up errors is not perceived as important, more valuable help is sought also from hierarchical equals implies a new nuance to this established finding: in a supportive climate, social barriers between equals are also lowered. In a non-punitive climate, hierarchical equals may be more willing to share relevant information and more willing to help a colleague, rather than viewing their colleague as a competitor.

To conclude, we find that young professionals in this study sought the most valuable help from hierarchical superiors, followed by individuals on the same hierarchical level, with accessibility playing a smaller, yet significant role. In a supportive learning from error climate, the learning value of help sought increases for both hierarchical superiors and for hierarchical equals: the social environment is therefore essential for overcoming barriers to help-seeking as a means of learning from errors.

\section{Limitations and Directions for Future Research}

This study is subject to several limitations and leads to questions for future research. First, this study was conducted with young professionals; therefore, the current sample does not allow us to take into account effects of task and error experience. As a consequence, several characteristics of the current sample need to be considered when attempting transfer to a different setting. First, junior auditors possess a master's degree in accountancy and build up work experience in a framework of structured on-the-job training. This body of knowledge means they are not complete novices when starting their audit work. Second, participants work under close supervision by a senior in a hierarchical team setting with multiple layers of reviews performed on their work. On the one hand, this provides a social structure for help seeking, on the other, this structure is indicative of error avoidance in the profession. Third, due to the focus on operative tasks at the junior level, and more strategic tasks at the senior level, hierarchical superiors are essential for addressing errors in order to ensure a highquality audit. For future research, it would be interesting to observe how help-seeking behavior changes after experiencing errors of different magnitudes. For example, we included two control questions in the survey: whether participants had made an error without serious consequences, and with serious consequences. We find negative correlations between making non-consequential errors and seeking help from colleagues of a higher hierarchical level $(r=-0.326, p<0.001)$, as well as between 
making consequential errors and the learning value of help received $(r=-0.163$, $p<0.050$ ). Including an interaction between error magnitude and perceptions of the firm's error climate could provide further evidence for the influence of climate on learning behavior.

Second, we make use of a non-situated measure in which participants look back at a personal experience through the ego-centric social network approach. This approach is established to be valuable in situations where direct observation is not possible (in this case due to privacy concerns of the part of the audit firms where client data are protected by law), as long as a clear prompt is presented and participants are asked about a specific behavior (Hatala 2006; Hatala and Fleming 2007). If the context allows, future research may use direct observations, reflective interventions implemented given a specific experience and even sociometric badges (see white papers by Choudhury and Pentland).

Third, this study focuses on young professionals within different organizations, but a single professional environment: auditing. This choice was made for two specific reasons. Auditing is particularly sensitive to errors and therefore a context in which meaningful data can be captured. Additionally, to reduce noise but maximize variance, young professionals from eleven different audit firms are included in this study. By focusing on one profession, generalizability of findings is limited. For example, the importance of hierarchy in audit teams may result in specific outcomes less applicable to more egalitarian contexts. Additionally, the hierarchical setting in auditing is associated with a clear division of tasks. In contexts where tasks are not split between operational and review, the relationship between professionals of different hierarchical levels may vary due to their respective knowledge, experiences and responsibilities. By implication, the social barriers may also vary, and the different mechanisms may be at work in overcoming these barriers, such as the knowledge and recognition of different expertise. Future research may establish whether the interaction between relative hierarchy and learning from error climate differs between settings in which hierarchy holds different relevance.

Fourth, we did not include a performance measure, which would be a relevant next step in understanding the long-term effects of learning from errors on performance, e.g. through a longitudinal design.

\section{Implications for Practice}

The findings of this study have several implications for organizations that want to encourage help-seeking as a form of learning from errors. These implications concern organizations' structuring of work as well as leader behavior. At the level of the organizations' structure, two implications become apparent: the creation of a supportive learning from error climate, and the design of work teams for the development of effective help-seeking networks. Putz et al. (2012) highlight that a learning from error climate can exist at several levels, from an entire organization and work groups to small teams and sub-groups of teams. A consistent climate across units within one organization therefore requires a structure of work in a way that supports learning from errors. Putz et al. (2012) review focuses for example on competition as a limiting factor of learning from errors, as it creates incentives to hide information and to engage in impression management, which makes the sharing of errors and error-derived 
knowledge less likely. Additionally, the authors stress the importance of formal and informal moments in which professionals have the opportunity to speak up and to share. Both of these mechanisms can be managed from the top of the organization. Moreover, at this level, team composition and project assignments are decided at high levels of an organization. By assigning teams with the development of effective help networks in mind, allocation decisions should consider (1) opportunities for the development and extension of (young) professional help networks, (2) opportunities for crossing hierarchical boundaries to maximize the diversity of help that can be sought, and (3) providing professionals sufficient time and opportunity to collaborate in order to increase accessibility of help sources. Finally, an organization's top can play a crucial role in deciding how the making of errors is handled. Incentives may be created to emphasize the prevention of errors, e.g. through punishments, or on learning, by rewarding the sharing of knowledge gained from error and providing resources for learning. The creation of the actual learning from error climate and the fostering of help-seeking behavior however may not be controlled from the top, but rather depend on behaviors of leaders throughout an organization. Edmondson (2011) emphasizes the crucial role of managers in shaping a firm's learning climate. By admitting own errors, offering support and resources for resolving and learning from errors, and by acknowledging the reporting and knowledge gained from learning from errors, supervisors can lead by example and set the tone for a supportive learning climate (Edmondson 2011; Putz et al. 2012). This essentially gives not just leaders, but also young professionals the language to discuss errors constructively and without assigning blame. In order to do so, leaders must not only honestly value learning and be prepared to invest time and energy in managing it, but they must also possess the skills to build personal relationships, to effectively communicate their values, to provide effective support, such as help and feedback, and they need to be competent in guiding their subordinates in proactively engaging in learning behavior after errors. This ability is not a trait and does not arise automatically with leader experience - it is the consequence of a work environment in which learning is organically integrated with daily practice and daily social interactions.

\section{Compliance with Ethical Standards}

Conflict of Interest This study has not received specific funding, the authors therefore declare no conflict of interest. All research has been conducted in compliance with ethical standards at Maastricht University.

Open Access This article is distributed under the terms of the Creative Commons Attribution 4.0 International License (http://creativecommons.org/licenses/by/4.0/), which permits unrestricted use, distribution, and reproduction in any medium, provided you give appropriate credit to the original author(s) and the source, provide a link to the Creative Commons license, and indicate if changes were made.

\section{References}

Abdolmohammadi, M. (1999). A comprehensive taxonomy of audit task structure, professional rank and decision aids for Behavioural research. Behavioral Research in Accounting, 11, 51-92.

Anseel, F., Lievens, F., \& Schollaert, E. (2009). Reflection as a strategy to enhance task performance after feedback. Organizational Behavior and Human Decision Processes, 110, 23-35. 
Ashford, S. J., \& Cummings, L. L. (1985). Proactive feedback seeking: The instumental use of the information environment. Journal of Occupational Psychology, 58, 67-79. https://doi.org/10.1111/j.2044-8325.1985. tb00181.x.

Bamberger, P. (2009). Employee help-seeking: Antecedents, consequences and new insights for future research. In J. J. Martocchio \& H. Liao (Eds.), Research on Personnel and Human Resource Management (Vol. 28, pp. 49-98). Emerald Group Publishing Limited.

Bauer, J., \& Mulder, R. H. (2013). Engagement in learning after errors at work: Enabling conditions and types of engagement. Journal of Education and Work, 26(1), 99-119. https://doi.org/10.1080 /13639080.2011.573776.

Bauer, J., Gartmeier, M., \& Harteis, C. (2012). Human fallibility and learning from errors at work. In J. Bauer \& C. Harteis (Eds.), Human fallibility. The ambiguity of errors for work and learning (pp. 155-170). Dordrecht: Springer.

Billett, S. (2012). Error and learning from errors at work. In J. Bauer \& C. Harteis (Eds.), Human Fallability the ambiguity of errors for work and learning (pp. 17-32). Dordrecht: Springer.

Borgatti, S. P., \& Cross, R. (2003). A relational view of information seeking and learning in social networks. Management Science, 49(4), 432-445. https://doi.org/10.1287/mnsc.49.4.432.14428.

Cannon, M. D., \& Edmondson, A. C. (2005). Failing to learn and learning to fail (Intelligently). Long Range Planning, 38(3), 299-319.

Carmeli, A., \& Gittell, J. H. (2009). High-quality relationships, psychological safety, and learning from failures in work organizations. Journal of Organizational Behaviour, 30, 709-729. https://doi. org/10.1002/job.

Catino, M., \& Patriotta, G. (2013). Learning from errors: Cognition, emotions and safety culture in the Italian air force. Organization Studies, 34(4), 437-467. https://doi.org/10.1177/0170840612467156.

Cattaneo, A. A. P., \& Boldrini, E. (2017). You learn by your mistakes. Effective training strategies based on the analysis of video-recorded worked-out examples. Vocations and Learning, 10(1), 1-26.

Cha, S. E., \& Edmondson, A. C. (2006). When values backfire: Leadership, attribution, and disenchantment in a values-driven organization. The Leadership Quarterly, 17(1), 57-78. https://doi.org/10.1016/j. leaqua.2005.10.006.

Cross, R., \& Sproull, L. (2004). More than an answer: Information relationships for actionable knowledge. Organization Science, 15(4), 446-462. https://doi.org/10.1287/orsc.1040.0075.

Edmondson, A. C. (2004). Learning from mistakes is easier said than done - group and organizational influences on the detection and correction of human error. The Journal of Applied Behavioral Science, 40(66), 66-90. https://doi.org/10.1177/0021886304263849.

Edmondson, A. C. (2011). Strategies for learning from failure. Harvard Business Review, (April), 48-55.

Edmondson, A. C. (2012). Teaming. In How organizations learn, innovate, and compete in the knowledge economy. San Francisco: Jossey-Bass.

Eraut, M. (2004). Informal learning in the workplace. Studies in Continuing Education, 26(2), 37-41. https://doi.org/10.1080/158037042000225245.

Eraut, M. (2007). Learning from other people in the workplace. Oxford Review of Education, 33(4), 403-422. https://doi.org/10.1080/03054980701425706.

Frese, M., \& Keith, N. (2015). Action errors, error management, and learning in organizations. Annual Review of Psychology, 66, 661-687. https://doi.org/10.1146/annurev-psych-010814-015205.

Gold, A., Gronewold, U., \& Salterio, S. E. (2014). Error management in audit firms: Error climate, type, and originator. Accounting Review, 89(1), 303-330. https://doi.org/10.2308/accr-50592.

Gronewold, U., \& Donle, M. (2011). Organizational error climate and auditors' predispositions toward handling errors. Behavioral Research in Accounting, 23(2), 69-92. https://doi.org/10.2308/bria-10061.

Gronewold, U., Gold, A., \& Salterio, S. E. (2013). Reporting self-made errors: The impact of organizational error-management climate and error type. Journal of Business Ethics, 117(1), 189-208. https://doi. org/10.1007/s10551-012-1500-6.

Harteis, C., Bauer, J., \& Haltia, P. (2007). Learning from errors in the workplace - insights from two studies in Germany and Finland. In H. Gruber \& T. Palonen (Eds.), Learning in the workplace - new developments (pp. 119-138). Turku: Finnish Education Research Association.

Hatala, J. P. (2006). Social network analysis in human resource development: A new methodology. Human Resource Development Review, 5(1), 45-71. https://doi.org/10.1177/1534484305284318.

Hatala, J. P., \& Fleming, P. (2007). Making transfer climate visible: Utilizing social network analysis to facilitate transfer of training. Human Resource Development Review, 6(1), 33-63. https://doi.org/10.1177 /1534484306297116.

Heck, R. H., Thomas, S. L., \& Tabata, L. N. (2014). Multilevel and longitudinal modeling with IBM SPSS (2nd ed.). Hove, East Sussex: Routledge. 
Helmreich, R. L. (2000). On error management : Lessons from aviation. British Medical Journal, 320(7237), 781-785. https://doi.org/10.1136/bmj.320.7237.781.

Hetzner, S., Gartmeier, M., Heid, H., \& Gruber, H. (2011). Error orientation and reflection at work. Vocations and Learning, 4, 25-39. https://doi.org/10.1007/s12186-010-9047-0.

Hofmann, D. A., Lei, Z., \& Grant, A. M. (2009). Seeking help in the shadow of doubt : The Sensemaking processes underlying how nurses decide whom to ask for advice. Journal of Applied Psychology, 94(5), 1261-1274. https://doi.org/10.1037/a0016557.

Jenkins, J. G., Deis, D. R., Bedard, J. C., \& Curtis, M. B. (2008). Accounting firm culture and governance: A research synthesis. Behavioral Research in Accounting, 20(1), 45-74. https://doi.org/10.2308 /bria.2008.20.1.45.

Lee, F. (1997). When the going gets tough, do the tough ask for help? Help seeking and power motivation in organizations. Organizational Behavior and Human Decision Processes, 72(3), 336-363. https://doi. org/10.1006/obhd.1997.2746.

Leicher, V., Mulder, R. H., \& Bauer, J. (2013). Learning from errors at work : A replication study in elder care nursing. Vocations and Learning, 6, 207-220. https://doi.org/10.1007/s12186-012-9090-0.

Lewis, K. (2004). Knowledge and performance in knowledge-worker teams: A longitudinal study of Transactive memory systems. Management Science, 50(11), 1519-1533. https://doi.org/10.1287 /mnsc.1040.0257.

Marsick, V. J., \& Volpe, M. (1999). The nature and need for informal learning. In V. J. Marsick \& M. Volpe (Eds.), Informal learning on the job (pp. 1-9). San Francisco, CA: Berrett Kohler.

Metcalfe, J. (2017). Learning from errors. Annual Review of Psychology, 68, 6.1-6.26. https://doi.org/10.2307 /20493670.

Nadler, A., Ellis, S., \& Bar, I. (2003). To seek or not to seek: The relationship between help seeking and job performance evaluations as moderated by task-relevant expertise. Journal of Applied Social Psychology, 33(1), 91-109. https://doi.org/10.1111/j.1559-1816.2003.tb02075.x.

Owhoso, V. E., Messier, W. F., \& Lynch, J. G. (2002). Error detection by industry-specialized teams during sequential audit review. Journal of Accounting Literature, 40(3), 883-900. https://doi.org/10.1111/1475679X.00075.

Perreault, S., Wainberg, J., \& Luippold, B. J. (2012). Does error-management climate impact auditor reporting decisions? An Investigation into the Inter-Organizational Effects of Tone-at-the-Top.

Plumlee, R. D., Rixom, B. a., \& Rosman, A. J. (2015). Training auditors to perform analytical procedures using metacognitive skills. The Accounting Review, 90(1), 351-369. https://doi.org/10.2308/accr-50856.

Putz, D., Schilling, J., \& Kluge, A. (2012). Measuring organizational climate for learning from errors at work. In J. Bauer \& C. Harteis (Eds.), Human Fallability-the ambiguity of errors for work and learning (pp. 107-123). Dordrecht: Springer.

Rybowiak, V., Garst, H., Frese, M., \& Batinic, B. (1999). Error orientation questionnaire (EOQ ): Reliability, validity, and different language equivalents. Journal of Organizational Behaviour, 20(4), 527-547. https://doi.org/10.1002/(SICI)1099-1379(199907)20:4<527::AID-JOB886>3.0.CO;2-G.

Scott, J. (2013). Social network analysis (3rd ed.). London: SAGE Publications.

Shute, V. J. (2008). Focus on formative feedback. Review of Educational Research, 78(1), 153-189.

Sibbald, M., de Bruin, A. B. H., \& van Merrienboer, J. J. G. (2013). Checklists improve experts' diagnostic decisions. Medical Education, 47(3), 301-308. https://doi.org/10.1111/medu.12080.

Stefaniak, C., \& Robertson, J. C. (2010). When auditors err: How mistake significance and superiors' historical reactions influence auditors' likelihood to admit a mistake. International Journal of Auditing, 14(1), 41-55. https://doi.org/10.1111/j.1099-1123.2009.00402.x.

Trotman, K. T., Bauer, T. D., \& Humphreys, K. A. (2015). Group judgment and decision making in auditing: Past and future research. Accounting, Organizations and Society, 47, 56-72. https://doi.org/10.1016/j. aos.2015.09.004.

Tucker, A., \& Edmondson, A. C. (2003). Why hospitals don't learn from failures: Organizational and psychological dynamics that inhibit system change. California Management Review, 45(2), 55-72. https://doi.org/10.2307/41166165.

Tynjälä, P. (2013). Toward a 3-P model of workplace learning: A literature review. Vocations and Learning, 6(1), 11-36. https://doi.org/10.1007/s12186-012-9091-z.

Van Der Kleij, R., \& Hoeppermans, M. (2011). How performance feedback and reflection affect transactive memory. In Proceedings of the Human Factors and Ergonomics Society (pp. 311-315). https://doi. org/10.1177/1071181311551065.

Van Der Rijt, J., \& Bossche, P. Van Den. (2013). Asking for help : A relational perspective on help seeking in the workplace, 259-279. https://oi.org/10.1007/s12186-012-9095-8. 
van der Rijt, J., van den Bossche, P., van de Wiel, M. W. J., de Maeyer, S., Gijselaers, W. H., \& Segers, M. S. R. (2013). Asking for help: A relational perspective on help seeking in the workplace. Vocations and Learning, 6(2), 259-279. https://doi.org/10.1007/s12186-012-9095-8.

van Duijnen, M. A. J., Van Busschbach, J. T., \& Snijders, T. A. B. (1999). Multilevel analysis of personal networks as dependent variables. Social Networks, 21, 187-209. https://doi.org/10.1016/S0378-8733(99)00009-X.

van Dyck, C., Frese, M., Baer, M., \& Sonnentag, S. (2005). Organizational error management culture and its impact on performance: A two-study replication. The Journal of Applied Psychology, 90(6), 1228-1240. https://doi.org/10.1037/0021-9010.90.6.1228 .

Wegner, D. M. (1987). Transactive memory: A contemporary analysis of the group mind. In M. B \& G. G. R (Eds.), Theories of group behavior. Springer series in social psychology (pp. 185-208). New York, NY: Springer. https://doi.org/10.1007/978-1-4612-4634-3_9.

Westermann, K. D., Bedard, J. C., \& Earley, C. E. (2015). Learning the "craft" of auditing: A dynamic view of auditors' on-the-job learning. Contemporary Accounting Research, 32(3), 864-896. https://doi. org/10.1111/1911-3846.12107.

Wolfe Morrison, E. (1993). Longitudinal study of the effects of information seeking on newcomer socialization. Journal of Applied Psychology, 78(2), 173-183. https://doi.org/10.1037//0021-9010.78.2.173.

Wolfe Morrison, E. (2012). Newcomer information Seeking: Exploring types, modes, sources, and outcomes. The Academy of Management Journal, 36(3), 557-589. https://doi.org/10.2307/256592 .

Zhao, B. (2011). Learning from errors : The role of context, emotion, and personality. Journal of Organizational Behavior, 463(June 2010), 435-463. https://doi.org/10.1002/job.

Zhao, B., \& Olivera, F. (2006). Error reporting in organizations. Academy of Management Review, 31(4), 1012-1030.

Therese Grohnert, Department of Educational Research and Development, School of Business and Economics, Maastricht University, the Netherlands. Therese Grohnert is post-doctoral researcher at the Department of Educational Research and Development at Maastricht University School of Business and Economics, email: t.grohnert@maastrichtuniversity.nl. Her research interests include workplace learning, especially from errors, expertise development, as well as judgment and decision-making, especially overconfidence.

Roger H. G. Meuwissen , Accounting \& Information Management, School of Business and Economics, Maastricht University, the Netherlands. Roger Meuwissen is Full Professor of Control and Auditing at Maastricht University School of Business and Economics; email r.meuwissen@maastrichtuniversity.nl. He is the author of several articles in academic journals and co-author of several textbooks on Internal Control and Accounting Information Systems. Currently, he is also a member of the Editorial Board of Accounting Education and director of the Maastricht Accounting, Auditing \& Information Management Research Center (MARC).

Wim H. Gijselaers , Department of Educational Research and Development, School of Business and Economics, Maastricht University, the Netherlands. Wim H. Gijselaers is Full Professor of Educational Research in the Department of Educational Research and Development at the School of Business and Economics of Maastricht University; email w.gijselaers@maastrichtuniversity.nl. His research focuses on professional development, focusing on sharing expertise within teams, and expertise development in business. He received his $\mathrm{PhD}$ in Education from Maastricht University. Currently, he is chief-editor of the Springer Book Series Innovation and Change in Professional Education. 